\title{
ON THE MAXIMAL OPERATORS OF T MEANS WITH RESPECT TO WALSH-KACZMARZ SYSTEM
}

\author{
NATA GOGOLASHVILI AND GEORGE TEPHNADZE
}

\begin{abstract}
In this paper we prove and discuss some new $\left(H_{p}, L_{p, \infty}\right)$ type inequalities of the maximal operators of $T$ means with monotone coefficients with respect to Walsh-Kaczmarz system. It is also proved that these results are the best possible in a special sense. As applications, both some well-known and new results are pointed out. In particular, we apply these results to prove a.e. convergence of such $T$ means.
\end{abstract}

2010 Mathematics Subject Classification. 42C10.

Key words and phrases: Walsh-Kaczmarz system, $T$ means, martingale Hardy space.

\section{INTRODUCTION}

Concerning definitions and notations used in this introduction we refer to Sections 2.

In 1948, S̆neider [37] introduced the Walsh-Kaczmarz system and showed that the inequality $\lim \sup _{n \rightarrow \infty} D_{n}^{\kappa}(x) / \log n \geq C>0$ holds a.e. In 1974 Schipp [32] and Young [47] proved that the Walsh-Kaczmarz system is a convergence system.

In [17] Goginava and Nagy proved that the maximal operator $R^{*, \kappa}$ of Riesz means with respect to Walsh-Kaczmarz system is bounded from the Hardy space $H_{1 / 2}$ to the space $L_{1 / 2, \infty}$, but is not bounded from the Hardy space $H_{p}$ to the space $L_{p}$, when $0<p \leq 1 / 2$. In [38] it was proved that there exists a martingale $f \in H_{p},(0<p \leq 1)$, such that the maximal operator $L^{*, \kappa}$ of Nörlund logarithmic means with respect to Walsh-Kaczmarz system is not bounded in the Lebesgue space $L_{p}$. The Logarithmic means with respect to the Walsh and Vilenkin systems systems were studied by Blahota and Gát [4], Gát 8], Lukkassen, Persson, Tephnadze and Tutberidze [14] (see also [13], [29], [31], 42], 44] and [46]), Simon [35].

In 1981, Skvortsov [36] showed that the Fejér means with respect to the Walsh-Kaczmarz system converge uniformly to $f$ for any continuous functions $f$. Gát [9] proved that, for any integrable functions, the Fejér means with respect to the Walsh-Kaczmarz system converges almost everywhere to the function. He showed that the maximal operator $\sigma^{*, \kappa}$ of WalshKaczmarz-Fejér means is of weak type $(1,1)$ and of type $(p, p)$ for all $1<p \leq \infty$. Gát's result was generalized by Simon [34], who showed that the maximal operator $\sigma^{*, \kappa}$ is of type $\left(H_{p}, L_{p}\right)$ for $p>1 / 2$. In the endpoint case $p=1 / 2$ Goginava [1] (see also [30, 39, 40, 41]) proved that maximal operator $\sigma^{*, \kappa}$ of Walsh-Kaczmarz-Fejér means is not of type $\left(H_{1 / 2}, L_{1 / 2}\right)$. Weisz [50] showed that the following is true:

Theorem W1. The maximal operator $\sigma^{*, \kappa}$ of Walsh-Kaczmarz-Fejér means is bounded from the Hardy space $H_{1 / 2}$ to the space $L_{1 / 2, \infty}$.

Gát and Goginava [10] proved that the maximal operator $\sigma^{\alpha, *, \kappa}$ of $(C, \alpha)(0<\alpha<1)$ means with respect Walsh-Kaczmarz system is bounded from the Hardy space $H_{1 /(1+\alpha)}$

The research was supported by Shota Rustaveli National Science Foundation grant no. FR-19-676. 
to the space $L_{1 /(1+\alpha), \infty}$. Goginava and Nagy [12 proved that $\sigma^{\alpha, *, \kappa}$ is not bounded from the Hardy space $H_{1 /(1+\alpha)}$ to the space $L_{1 /(1+\alpha)}$. Móricz and Siddiqi [19] investigated the approximation properties of some special Nörlund means of $L_{p}$ function in norm. These means in the martingale Hardy spaces were discussed in Blahota, Tephnadze [5, 6]. In [28] and [43] (see also [1, 7, 16]) it was proved some $\left(H_{p}, L_{p}\right)$-type inequalities for the maximal operators of Nörlund means, with respect to Walsh-Kaczmarz and Vilenkin systems, when $0<p \leq 1$. In the two dimensional case approximation properties of Nörlund and Cesáro means were considered by Nagy [20, 21, 22] and by Nagy and Tephnadze [23, 24, 25, 26, 27]. Some boundedness results of so called $T, \Theta$ and $\theta$ means in the Lebesgue and martingale Hardy spaces can be found in Blahota and Nagy [2], in Blahota, Nagy and Tephnadze [3], Tutberidze [45] and Weisz [51, 52, 53, 54].

The main aim of this paper is to investigate $\left(H_{p}, L_{p, \infty}\right)$-type inequalities for the maximal operators of $T$ means with monotone coefficients of the one-dimensional Kaczmarz-Fourier series.

This paper is organized as follows: in order not to disturb our discussions later on some definitions and notations are presented in Section 2. The main results and some of its consequences can be found in Section 3. For the proofs of the main results we need some auxiliary results of independent interest. Also these results are presented in Section 3. The detailed proofs are given in Section 4.

\section{Definitions and Notations}

Now, we give a brief introduction to the theory of dyadic analysis [33]. Let $\mathbf{N}_{+}$denote the set of positive integers, $\mathbf{N}:=\mathbf{N}_{+} \cup\{0\}$.

Denote $\mathbb{Z}_{2}$ the discrete cyclic group of order 2 , that is $\mathbb{Z}_{2}=\{0,1\}$, where the group operation is the modulo 2 addition and every subset is open. The Haar measure on $\mathbb{Z}_{2}$ is given such that the measure of a singleton is $1 / 2$. Let $G$ be the complete direct product of the countable infinite copies of the compact groups $\mathbb{Z}_{2}$. The elements of $G$ are of the form

$$
x=\left(x_{0}, x_{1}, \ldots, x_{k}, \ldots\right), \quad x_{k}=0 \vee 1,(k \in \mathbf{N}) .
$$

The group operation on $G$ is the coordinate-wise addition, the measure (denoted by $\mu$ ) and the topology are the product measure and topology. The compact Abelian group $G$ is called the Walsh group. A base for the neighborhoods of $G$ can be given in the following way:

$$
I_{0}(x):=G, \quad I_{n}(x):=I_{n}\left(x_{0}, \ldots, x_{n-1}\right):=\left\{y \in G: y=\left(x_{0}, \ldots, x_{n-1}, y_{n}, y_{n+1}, \ldots\right)\right\},
$$

$(x \in G, n \in \mathbf{N})$. These sets are called dyadic intervals. Denote by $0=(0: i \in \mathbf{N}) \in G$ the null element of $G$. Let $I_{n}:=I_{n}(0), \overline{I_{n}}:=G \backslash I_{n}(n \in \mathbf{N})$. Set $e_{n}:=(0, \ldots, 0,1,0, \ldots) \in G$, the $n$-th coordinate of which is 1 and the rest are zeros $(n \in \mathbf{N})$.

For $k \in \mathbf{N}$ and $x \in G$ let us denote the $k$-th Rademacher function, by

$$
r_{k}(x):=(-1)^{x_{k}} .
$$

Now, define the Walsh system $w:=\left(w_{n}: n \in \mathbf{N}\right)$ on $G$ as:

$$
w_{n}(x):=\prod_{k=0}^{\infty} r_{k}^{n_{k}}(x)=r_{|n|}(x)(-1) \sum_{k=0}^{|n|-1} n_{k} x_{k} \quad(n \in \mathbf{N}) .
$$


If $n \in \mathbf{N}$, then $n=\sum_{i=0}^{\infty} n_{i} 2^{i}$ can be written, where $n_{i} \in\{0,1\} \quad(i \in \mathbf{N})$, i. e. $n$ is expressed in the number system of base 2. Denote $|n|:=\max \left\{j \in \mathbf{N}: n_{j} \neq 0\right\}$, that is $2^{|n|} \leq n<2^{|n|+1}$.

The Walsh-Kaczmarz functions are defined by

$$
\kappa_{n}(x):=r_{|n|}(x) \prod_{k=0}^{|n|-1}\left(r_{|n|-1-k}(x)\right)^{n_{k}}=r_{|n|}(x)(-1) \sum_{k=0}^{|n|-1} n_{k} x_{|n|-1-k} .
$$

The Dirichlet kernels are defined by

$$
D_{0}:=0, \quad D_{n}^{\psi}:=\sum_{i=0}^{n-1} \psi_{i}, \quad(\psi=w \text { or } \psi=\kappa) .
$$

The $2^{n}$-th Dirichlet kernels have a closed form (see e.g. [33])

$$
D_{2^{n}}^{w}(x)=D_{2^{n}}(x)=D_{2^{n}}^{\kappa}(x)= \begin{cases}2^{n} & x \in I_{n}, \\ 0 & x \notin I_{n} .\end{cases}
$$

The norm (or quasi-norm) of the spaces $L_{p}(G)$ and $L_{p, \infty}(G)$ are respectively defined by

$$
\|f\|_{p}^{p}:=\int_{G}|f|^{p} d \mu, \quad\|f\|_{L_{p, \infty}(G)}^{p}:=\sup _{\lambda>0} \lambda^{p} \mu(f>\lambda), \quad(0<p<\infty) .
$$

The partial sums with respect to Walsh and Walsh-Kaczmarz series are defined as follows:

$$
S_{M}^{\psi} f:=\sum_{i=0}^{M-1} \widehat{f}(i) \psi_{i}, \quad(\psi=w \text { or } \psi=\kappa) .
$$

Let $\left\{q_{k}: k \geq 0\right\}$ be a sequence of nonnegative numbers. The $n$-th Nörlund and $T$ means for a Fourier series of $f$ are respectively defined by

$$
t_{n}^{\psi} f=\frac{1}{Q_{n}} \sum_{k=1}^{n} q_{n-k} S_{k}^{\psi} f, \quad(\psi=w \text { or } \psi=\kappa)
$$

and

$$
T_{n}^{\psi} f:=\frac{1}{Q_{n}} \sum_{k=0}^{n-1} q_{k} S_{k}^{\psi} f, \quad(\psi=w \text { or } \psi=\kappa) .
$$

where $Q_{n}:=\sum_{k=0}^{n-1} q_{k}$. It is obvious that

$$
T_{n}^{\psi} f(x)=\int_{G_{m}} f(t) F_{n}^{\psi}(x-t) d \mu(t)
$$

where $F_{n}^{\psi}:=\frac{1}{Q_{n}} \sum_{k=0}^{n-1} q_{k} D_{k}^{\psi}$ is called $T$ kernel.

We always assume that $\left\{q_{k}: k \geq 0\right\}$ be a sequence of nonnegative numbers and $q_{0}>0$. Then the summability method (2) generated by $\left\{q_{k}: k \geq 0\right\}$ is regular if and only if $\lim _{n \rightarrow \infty} Q_{n}=\infty$. 
Let consider some class of $T$ means with monotone and bounded sequence $\left\{q_{k}: k \in \mathbb{N}\right\}$, such that

$$
q:=\lim _{n \rightarrow \infty} q_{n}>c>0
$$

Then, it easy to check that

$$
\frac{q_{n-1}}{Q_{n}}=O\left(\frac{1}{n}\right), \text { as } n \rightarrow \infty .
$$

The $n$-th Fejér means of a function $f$ is given by

$$
\sigma_{n}^{\psi} f:=\frac{1}{n} \sum_{k=0}^{n-1} S_{k}^{\psi} f, \quad(\psi=w \text { or } \psi=\kappa) .
$$

Fejér kernel is defined in the usual manner

$$
K_{n}^{\psi}:=\frac{1}{n} \sum_{k=1}^{n} D_{k}^{\psi}, \quad(\psi=w \text { or } \psi=\kappa) .
$$

If we invoke Abel transformation to obtain the following identities, which are very important for the investigations of $T$ summability:

$$
Q_{n}:=\sum_{j=0}^{n-1} q_{j}=\sum_{j=0}^{n-2}\left(q_{j}-q_{j+1}\right) j+q_{n-1}(n-1)
$$

and

$$
F_{n}^{\psi}=\frac{1}{Q_{n}}\left(\sum_{j=0}^{n-2}\left(q_{j}-q_{j+1}\right) j K_{j}^{\psi}+q_{n-1}(n-1) K_{n-1}^{\psi}\right) .
$$

The $(C, \alpha)$-means are defined as

$$
\sigma_{n}^{\alpha, \psi} f=\frac{1}{A_{n}^{\alpha}} \sum_{k=1}^{n} A_{n-k}^{\alpha-1} S_{k}^{\psi} f, \quad(\psi=w \text { or } \psi=\kappa),
$$

where

$$
A_{0}^{\alpha}=0, \quad A_{n}^{\alpha}=\frac{(\alpha+1) \ldots(\alpha+n)}{n !}, \alpha \neq-1,-2, \ldots
$$

It is known that

$$
A_{n}^{\alpha} \sim n^{\alpha}, \quad A_{n}^{\alpha}-A_{n-1}^{\alpha}=A_{n}^{\alpha-1}, \sum_{k=1}^{n} A_{n-k}^{\alpha-1}=A_{n}^{\alpha} .
$$

We also consider "inverse" $(C, \alpha)$-means, which is example of $T$-means:

$$
U_{n}^{\alpha, \psi} f:=\frac{1}{A_{n}^{\alpha}} \sum_{k=0}^{n-1} A_{k}^{\alpha-1} S_{k}^{\psi} f, \quad(\psi=w \text { or } \psi=\kappa) .
$$

Let $\beta_{n}^{\alpha}$ denote the $T$ mean, where

$$
\left\{q_{0}=1, \quad q_{k}=k^{\alpha-1}: k \in \mathbb{N}_{+}\right\},
$$


that is

$$
V_{n}^{\alpha, \psi} f:=\frac{1}{Q_{n}} \sum_{k=1}^{n} k^{\alpha-1} S_{k}^{\psi} f, \quad(\psi=w \text { or } \psi=\kappa) \quad 0<\alpha<1 .
$$

The $n$-th Riesz's logarithmic mean $R_{n}^{\psi}$ and Nörlund logarithmic mean $L_{n}^{\psi}$ are defined by

$$
R_{n}^{\psi} f:=\frac{1}{l_{n}} \sum_{k=0}^{n-1} \frac{S_{k}^{\psi} f}{k}, \quad L_{n}^{\psi} f:=\frac{1}{l_{n}} \sum_{k=1}^{n-1} \frac{S_{k}^{\psi} f}{n-k}, \quad(\psi=w \text { or } \psi=\kappa) .
$$

respectively, where $l_{n}:=\sum_{k=1}^{n-1} 1 / k$.

Up to now we have considered $T$ mean in the case when the sequence $\left\{q_{k}: k \in \mathbb{N}\right\}$ is bounded but now we consider $T$ summabilities with unbounded sequence $\left\{q_{k}: k \in \mathbb{N}\right\}$. Let $\alpha \in \mathbb{R}_{+}, \quad \beta \in \mathbb{N}_{+}$and

$$
\log (\beta) x:=\overbrace{\log \ldots \log x}^{\beta-\text { times }}
$$

If we define the sequence $\left\{q_{k}: k \in \mathbb{N}\right\}$ by

$$
\left\{q_{0}=0 \text { and } q_{k}=\log ^{(\beta)} k^{\alpha}: k \in \mathbb{N}_{+}\right\},
$$

then we get the class of $T$ means with non-decreasing coefficients:

$$
B_{n}^{\alpha, \beta, \psi} f:=\frac{1}{Q_{n}} \sum_{k=1}^{n} \log ^{(\beta)} k^{\alpha} S_{k}^{\psi} f, \quad(\psi=w \text { or } \psi=\kappa) .
$$

We note that $B_{n}^{\alpha, \beta}$ are well-defined for every $n \in \mathbb{N}$

$$
B_{n}^{\alpha, \beta, \psi} f=\sum_{k=0}^{n-1} \frac{\log ^{(\beta)} k^{\alpha}}{Q_{n}} S_{k}^{\psi} f, \quad(\psi=w \text { or } \psi=\kappa) .
$$

It is obvious that $\frac{n}{2} \log ^{(\beta)} \frac{n^{\alpha}}{2^{\alpha}} \leq Q_{n} \leq n \log ^{(\beta)} n^{\alpha}$. It follows that

$$
\frac{q_{n-1}}{Q_{n}} \leq \frac{c \log ^{(\beta)}(n-1)^{\alpha}}{n \log ^{(\beta)} n^{\alpha}}=O\left(\frac{1}{n}\right) \rightarrow 0, \text { as } n \rightarrow \infty .
$$

Let us define maximal operator of $T$ means by

$$
T^{*, \psi} f:=\sup _{n \in \mathbb{N}}\left|T_{n}^{\psi} f\right|, \quad(\psi=w \text { or } \psi=\kappa) .
$$

The well-known example of maximal operator of $T$ means are Fejer and Riesz logarithmic means which are defined by:

$$
\sigma^{*, \psi} f:=\sup _{n \in \mathbb{N}}\left|\sigma_{n}^{\psi} f\right|, \quad R^{*, \psi} f:=\sup _{n \in \mathbb{N}}\left|R_{n}^{\psi} f\right| \quad(\psi=w \text { or } \psi=\kappa)
$$

respectively. We also define some new maximal operators:

$U^{\alpha, \beta, *, \psi} f:=\sup _{n \in \mathbb{N}}\left|U_{n}^{\alpha, \beta, \psi} f\right|, V^{\alpha, *, \psi} f:=\sup _{n \in \mathbb{N}}\left|V_{n}^{\alpha, \psi} f\right|, B^{\alpha, *, \psi} f:=\sup _{n \in \mathbb{N}}\left|B_{n}^{\alpha, \psi} f\right|(\psi=w$ or $\psi=\kappa)$.

For the martingale $f$ we consider the following maximal operators of some Norlund means:

$$
t^{*, \psi} f:=\sup _{n \in \mathbb{N}}\left|t_{n}^{\psi} f\right|, \quad \sigma^{\alpha, *, \psi} f:=\sup _{n \in \mathbb{N}}\left|\sigma_{n}^{\alpha, \psi} f\right|, \quad L^{*, \psi} f:=\sup _{n \in \mathbb{N}}\left|L_{n}^{\psi} f\right|, \quad(\psi=w \text { or } \psi=\kappa) .
$$


The $\sigma$-algebra generated by the dyadic intervals of measure $2^{-k}$ will be denoted by $F_{k}$ $(k \in \mathbf{N})$. Denote by $f=\left(f^{(n)}, n \in \mathbf{N}\right)$ a martingale with respect to $\left(F_{n}, n \in \mathbf{N}\right)$ (for details see, e. g. [48, 49]). The maximal function of a martingale $f$ is defined by $f^{*}=\sup _{n \in \mathbf{N}}\left|f^{(n)}\right|$.

In case $f \in L_{1}(G)$, the maximal function can also be given by

$$
f^{*}(x)=\sup _{n \in \mathbf{N}} \frac{1}{\mu\left(I_{n}(x)\right)}\left|\int_{I_{n}(x)} f(u) d \mu(u)\right|, \quad x \in G .
$$

For $0<p<\infty$ the Hardy martingale space $H_{p}(G)$ consists of all martingales for which

$$
\|f\|_{H_{p}}:=\left\|f^{*}\right\|_{p}<\infty .
$$

If $f \in L_{1}(G)$, then it is easy to show that the sequence $\left(S_{2^{n}}^{\psi} f: n \in \mathbf{N}\right)$ is a martingale.

The Walsh-Fourier and Walsh-Kaczmarz-Fourier coefficients of $f \in L_{1}(G)$ are the same as the ones of the martingale $\left(S_{2^{n}}^{\psi} f: n \in \mathbf{N}\right)$ obtained from $f$.

If $f$ is a martingale, then the Walsh-Fourier and Walsh-Kaczmarz-Fourier coefficients must be defined in a little bit different way:

$$
\widehat{f}^{\psi}(i)=\lim _{n \rightarrow \infty} \int_{G} f^{(n)} \psi_{i} d \mu, \quad(\psi=w, \text { or } \psi=\kappa) .
$$

A bounded measurable function $a$ is p-atom, if there exists an interval $I$, such that

$$
\int_{I} a d \mu=0, \quad\|a\|_{\infty} \leq \mu(I)^{-1 / p}, \quad \operatorname{supp}(a) \subset I .
$$

Weisz proved that Hardy spaces $H_{p}$ have atomic characterization. In particular the following is true:

Proposition 1. 48 A martingale $f=\left(f^{(n)}, n \in \mathbb{N}\right)$ is in $H_{p}(0<p \leq 1)$ if and only if there exists sequence $\left(a_{k}, k \in \mathbb{N}\right)$ of p-atoms and a sequence $\left(\mu_{k}, k \in \mathbb{N}\right)$, of real numbers, such that, for every $n \in \mathbb{N}$,

$$
\sum_{k=0}^{\infty} \mu_{k} S_{2^{n}}^{\psi} a_{k}=f^{(n)}, \quad \sum_{k=0}^{\infty}\left|\mu_{k}\right|^{p}<\infty, \quad(\psi=w \text { or } \psi=\kappa) .
$$

Moreover,

$$
\|f\|_{H_{p}} \backsim \inf \left(\sum_{k=0}^{\infty}\left|\mu_{k}\right|^{p}\right)^{1 / p}
$$

where the infimum is taken over all decomposition of $f$ of the form (7).

Proposition 2. [48, 49] Suppose that an operator $T$ is $\sigma$-linear and for some $0<p<1$

$$
\|T f\|_{L_{p, \infty}} \leq c_{p}\|f\|_{H_{p}}
$$

then $T$ is of weak type- $(1,1)$ :

$$
\|T f\|_{L_{1, \infty}} \leq c\|f\|_{1} .
$$




\section{Results}

\section{Main results and some of its consequences}

We state our main result concerning the maximal operator of the summation method (2), which we also show is in a sense sharp.

Theorem 1. a) The maximal operator $T^{*, \kappa}$ of the summability method (2) with nonincreasing sequence $\left\{q_{k}: k \geq 0\right\}$, is bounded from the Hardy space $H_{1 / 2}$ to the space $L_{1 / 2, \infty}$.

The statement in a) is sharp in the following sense: b) Let $0<p<1 / 2$ and $\left\{q_{k}: k \geq 0\right\}$

is non-decreasing sequence, satisfying the condition

$$
\frac{q_{n+1}}{Q_{n+2}} \geq \frac{c}{n}, \quad(c \geq 1) .
$$

Then there exists a martingale $f \in H_{p}$, such that

$$
\sup _{n \in \mathbb{N}}\left\|T_{n}^{\kappa} f\right\|_{L_{p, \infty}}=\infty
$$

A number of special cases of our results are of particular interest and give both wellknown and new information. We just give the following examples of such $T$ means with non-increasing sequence $\left\{q_{k}: k \geq 0\right\}$ :

Corollary 1. The maximal operators $U^{\alpha, \beta, *, \kappa}, V^{\alpha, *, \kappa}$ and $R^{*, \kappa}$ are bounded from the Hardy space $H_{1 / 2}$ to the space $L_{1 / 2, \infty}$ but are not bounded from $H_{p}$ to the space $L_{p, \infty}$, when $0<p<$ $1 / 2$.

Corollary 2. Let $f \in L_{1}$ and $T_{n}^{\kappa}$ be the $T$ means with non-increasing sequence $\left\{q_{k}: k \geq 0\right\}$. Then $T_{n}^{\kappa} f \rightarrow f$, a.e., as $n \rightarrow \infty$.

Corollary 3. Let $f \in L_{1}$. Then

$$
\begin{aligned}
R_{n}^{\kappa} f & \rightarrow f, \quad \text { a.e., } \\
U_{n}^{\alpha, \beta, \kappa} f & \rightarrow f, \quad \text { as } n \rightarrow \infty, \\
V_{n}^{\alpha, \kappa} f & \rightarrow f, \quad \text { a.e., } \quad \text { as } n \rightarrow \infty,
\end{aligned}
$$

Theorem 2. a) The maximal operator $T^{*, \kappa}$ of the summability method (2) with non-decreasing sequence $\left\{q_{k}: k \geq 0\right\}$ satisfying the condition

$$
\frac{q_{n-1}}{Q_{n}}=O\left(\frac{1}{n}\right), \quad \text { as } n \rightarrow \infty,
$$

is bounded from the Hardy space $H_{1 / 2}$ to the space $L_{1 / 2, \infty}$.

Our next result shows that the statement in b) above hold also for non-decreasing sequences and now without any restriction like (8).

b) Let $0<p<1 / 2$. For any non-decreasing sequence $\left\{q_{k}: k \geq 0\right\}$, there exists a martingale $f \in H_{p}$, such that

$$
\sup _{n \in \mathbb{N}}\left\|T_{n}^{\kappa} f\right\|_{L_{p, \infty}}=\infty
$$

A number of special cases of our results are of particular interest and give both wellknown and new information. We just give the following examples of such $T$ means with non-decreasing sequence $\left\{q_{k}: k \geq 0\right\}$ : 
Corollary 4. The maximal operator $B^{\alpha, \beta, *, \kappa}$ is bounded from the Hardy space $H_{1 / 2}$ to the space $L_{1 / 2, \infty}$ but is not bounded from $H_{p}$ to the space $L_{p, \infty}$, when $0<p<1 / 2$.

Corollary 5. Let $f \in L_{1}$ and $T_{n}^{\kappa}$ be the $T$ means with non-decreasing sequence $\left\{q_{k}: k \geq 0\right\}$ and satisfying condition (9). Then

$$
T_{n}^{\kappa} f \rightarrow f, \quad \text { a.e., } \quad \text { as } n \rightarrow \infty \text {. }
$$

Corollary 6. Let $f \in L_{1}$. Then $B_{n}^{\alpha, \beta, \kappa} f \rightarrow f, \quad$ a.e., $\quad$ as $n \rightarrow \infty$.

\section{ProOFS}

Proof of Theorem 1. Let the sequence $\left\{q_{k}: k \geq 0\right\}$ be non-increasing. By combining (44) with (5) and using Abel transformation we get that

$$
\begin{aligned}
\left|T_{n}^{\kappa} f\right| & \leq\left|\frac{1}{Q_{n}} \sum_{j=1}^{n-1} q_{j} S_{j}^{\kappa} f\right| \\
& \leq \frac{1}{Q_{n}}\left(\sum_{j=1}^{n-2}\left|q_{j}-q_{j+1}\right| j\left|\sigma_{j}^{\kappa} f\right|+q_{n-1}(n-1)\left|\sigma_{n}^{\kappa} f\right|\right) \\
& \leq \frac{1}{Q_{n}}\left(\sum_{j=1}^{n-2}\left(q_{j}-q_{j+1}\right) j+q_{n-1}(n-1)\right) \sigma^{*, \kappa} f \leq \sigma^{*, \kappa} f
\end{aligned}
$$

so that

$$
T^{*, \kappa} f \leq \sigma^{*, \kappa} f
$$

If we apply (10) and Theorem W1 we can conclude that the maximal operators $T^{*, \kappa}$ of all $T$ means with non-increasing sequence $\left\{q_{k}: k \geq 0\right\}$, are bounded from the Hardy space $H_{1 / 2}$ to the space weak $-L_{1 / 2}$.

On the other hand by using proposition 2 we also have weak $(1,1)$ type inequality and by well-known density argument due to Marcinkiewicz and Zygmund [15] we have $T_{n}^{\kappa} f \rightarrow f$, a.e., for all $f \in L_{1}$.

Let $0<p<1 / 2$ and $\left\{\alpha_{k}: k \in \mathbb{N}\right\}$ be an increasing sequence of positive integers such that:

$$
\begin{gathered}
\sum_{k=0}^{\infty} 1 / \alpha_{k}^{p}<\infty, \\
\sum_{\eta=0}^{k-1} \frac{2^{\alpha_{\eta} / p}}{\alpha_{\eta}}<\frac{2^{\alpha_{k} / p-1}}{2 \alpha_{k}}, \\
\frac{2^{\alpha_{k-1}(1 / p-1)}}{\alpha_{k-1}}<\frac{2^{\alpha_{k}(1 / p-1)-4}}{\alpha_{k}} .
\end{gathered}
$$

We note that such an increasing sequence $\left\{\alpha_{k}: k \in \mathbb{N}\right\}$ which satisfies conditions (11-13) can be constructed. 
Let

$$
f^{(A)}=\sum_{\left\{k ; \lambda_{k}<A\right\}} \lambda_{k} a_{k}
$$

where

$$
\lambda_{k}=\frac{1}{\alpha_{k}} \quad \text { and } \quad a_{k}=2^{\alpha_{k}(1 / p-1)}\left(D_{2^{\alpha_{k}+1}}-D_{2^{\alpha_{k}}}\right) .
$$

By using Proposition 1, it is easy to see that the martingale $f=\left(f^{(1)}, f^{(2)} \ldots f^{(A)} \ldots\right) \in H_{1 / 2}$. Moreover, it is easy to show that

$$
\widehat{f}(j)= \begin{cases}\frac{2^{\alpha_{k}(1 / p-1)}}{\alpha_{k}}, & \text { if } j \in\left\{2^{\alpha_{k}}, \ldots, 2^{\alpha_{k}+1}-1\right\}, k=0,1,2 \ldots, \\ 0, & \text { if } j \notin \bigcup_{k=1}^{\infty}\left\{2^{\alpha_{k}}, \ldots, 2^{\alpha_{k}+1}-1\right\}\end{cases}
$$

We can write

$$
T_{2^{\alpha_{k}+2}}^{\kappa} f=\frac{1}{Q_{2^{\alpha_{k}}+2}} \sum_{j=0}^{2^{\alpha_{k}}} q_{j} S_{j}^{\kappa} f+\frac{q_{2^{\alpha_{k}}+1}}{Q_{2^{\alpha_{k}}+2}} S_{2^{\alpha_{k}+1}}^{\kappa} f:=I+I I .
$$

Let $2^{\alpha_{s}} \leq j \leq 2^{\alpha_{s}+1}$, where $s=0, \ldots, k-1$. Moreover,

$$
\left|D_{j}^{\kappa}-D_{2^{\alpha_{s}}}\right| \leq j-2^{\alpha_{s}} \leq 2^{\alpha_{s}}, \quad(s \in \mathbb{N})
$$

so that, according to (11) and (15), we have that

$$
\begin{aligned}
& \left|S_{j}^{\kappa} f\right|=\left|\sum_{v=0}^{2^{\alpha_{s-1}+1}-1} \widehat{f}(v) \kappa_{v}+\sum_{v=2^{\alpha_{s}}}^{j-1} \widehat{f}(v) \kappa_{v}\right| \\
\leq & \left|\sum_{\eta=0}^{s-1} \sum_{v=2^{\alpha_{\eta}}}^{2^{\alpha_{\eta}+1}-1} \frac{2^{\alpha_{\eta}(1 / p-1)}}{\alpha_{\eta}} \kappa_{v}\right|+\frac{2^{\alpha_{s}(1 / p-1)}}{\alpha_{s}}\left|\left(D_{j}^{\kappa}-D_{2^{\alpha_{s}}}\right)\right| \\
= & \left|\sum_{\eta=0}^{s-1} \frac{2^{\alpha_{\eta}(1 / p-1)}}{\alpha_{\eta}}\left(D_{2^{\alpha_{\eta}+1}}-D_{2^{\alpha_{\eta}}}\right)\right|+\frac{2^{\alpha_{s}(1 / p-1)}}{\alpha_{s}}\left|\left(D_{j}^{\kappa}-D_{2^{\alpha_{s}}}\right)\right| \\
\leq & \sum_{\eta=0}^{s-1} \frac{2^{\alpha_{\eta} / p}}{\alpha_{\eta}}+\frac{2^{\alpha_{s} / p}}{\alpha_{s}} \leq \frac{2^{\alpha_{s-1} / p+1}}{\alpha_{s-1}}+\frac{2^{\alpha_{s} / p}}{\alpha_{s}} \leq \frac{2^{\alpha_{k-1} / p+1}}{\alpha_{k-1}} .
\end{aligned}
$$

Let $2^{\alpha_{s-1}+1}+1 \leq j \leq 2^{\alpha_{s}}$, where $s=1, \ldots, k$. Analogously to (17) we can prove that

$$
\begin{aligned}
& \left|S_{j}^{\kappa} f\right|=\left|\sum_{v=0}^{2^{\alpha_{s-1}+1}-1} \widehat{f}(v) \psi_{v}\right|=\left|\sum_{\eta=0}^{s-1} \sum_{v=2^{\alpha_{\eta}}}^{2^{\alpha_{\eta}+1}-1} \frac{2^{\alpha_{\eta}(1 / p-1)}}{\alpha_{\eta}} \kappa_{v}\right| \\
= & \left|\sum_{\eta=0}^{s-1} \frac{M_{\alpha_{\eta}}^{1 / p-1}}{\alpha_{\eta}}\left(D_{2^{\alpha_{\eta}+1}}-D_{2^{\alpha_{\eta}}}\right)\right| \leq \frac{2^{\alpha_{s-1} / p+1}}{\alpha_{s-1}} \leq \frac{2^{\alpha_{k-1} / p+1}}{\alpha_{k-1}} .
\end{aligned}
$$

Hence,

$$
|I| \leq \frac{1}{Q_{2^{\alpha_{k}+2}}} \sum_{j=0}^{2^{\alpha_{k}}} q_{j}\left|S_{j}^{\kappa} f\right| \leq \frac{2^{\alpha_{k-1} / p+1}}{\alpha_{k-1}} \frac{1}{Q_{M_{\alpha_{k}}+1}} \sum_{j=0}^{2^{\alpha_{k}}} q_{j} \leq \frac{2^{\alpha_{k-1} / p+1}}{\alpha_{k-1}}
$$


If we now apply (15) and (17) we get that

$$
\begin{aligned}
|I I| & =\frac{q_{2^{\alpha_{k}+1}}}{Q_{2^{\alpha_{k}}+2}}\left|\frac{2^{\alpha_{k}(1 / p-1)}}{\alpha_{k}} \kappa_{2^{\alpha_{k}}}+S_{2^{\alpha_{k}}} f\right| \\
& =\frac{q_{2^{\alpha_{k}}+1}}{Q_{2^{n_{k}}+2}}\left|\frac{2^{\alpha_{k}(1 / p-1)}}{\alpha_{k}} \kappa_{2^{\alpha_{k}}}+S_{2^{\alpha_{k-1}+1}} f\right| \\
& \geq \frac{q_{2^{\alpha_{k}+1}}}{Q_{2^{n_{k}}+2}}\left(\left|\frac{2^{\alpha_{k}(1 / p-1)}}{\alpha_{k}} \kappa_{2^{\alpha_{k}}}\right|-\left|S_{2^{\alpha_{k-1}+1}} f\right|\right) \\
& \geq \frac{q_{2^{\alpha_{k}}+1}}{Q_{2^{\alpha_{k}}+2}}\left(\frac{2^{\alpha_{k}(1 / p-1)}}{\alpha_{k}}-\frac{2^{\alpha_{k-1} / p+2}}{\alpha_{k-1}}\right) \\
& \geq \frac{q_{2^{\alpha_{k}}+1}}{Q_{2^{\alpha_{k}}+2}} \frac{2^{\alpha_{k}(1 / p-1)-2}}{\alpha_{k}} .
\end{aligned}
$$

Without lost the generality we may assume that $c=1$ in (8). By combining (18) and (19) we get

$$
\begin{aligned}
\left|T_{2^{\alpha_{k+2}}}^{\kappa} f\right| & \geq|I I|-|I| \\
& \geq \frac{q_{2^{\alpha_{k}+1}}}{Q_{2^{\alpha_{k}+2}}} \frac{2^{\alpha_{k}(1 / p-1)-2}}{\alpha_{k}}-\frac{2^{\alpha_{k-1} / p+1}}{\alpha_{k-1}} \\
& \geq \frac{2^{\alpha_{k}(1 / p-2)}}{4 \alpha_{k}}-\frac{2^{\alpha_{k-1} / p+1}}{\alpha_{k-1}} \geq \frac{2^{\alpha_{k}(1 / p-2)}}{16 \alpha_{k}} .
\end{aligned}
$$

On the other hand,

$$
\mu\left\{x \in G_{m}:\left|T_{2^{\alpha_{k}+2}}^{\kappa} f(x)\right| \geq \frac{2^{\alpha_{k}(1 / p-2)}}{16 \alpha_{k}}\right\}=\mu\left(G_{m}\right)=1 .
$$

Let $0<p<1 / 2$. Then

$$
\begin{aligned}
& \frac{2^{\alpha_{k}(1 / p-2)}}{16 \alpha_{k}} \cdot\left(\mu\left\{x \in G_{m}:\left|T_{M_{\alpha_{k}}+2}^{\kappa} f(x)\right| \geq \frac{2^{\alpha_{k}(1 / p-2)}}{16 \alpha_{k}}\right\}\right)^{1 / p} \\
= & \frac{2^{\alpha_{k}(1 / p-2)}}{16 \alpha_{k}} \rightarrow \infty, \text { as } k \rightarrow \infty .
\end{aligned}
$$

The proof is complete.

Proof of Corollary 1. Since $R_{n}^{\kappa}, U_{n}^{\alpha, \kappa}$ and $V_{n}^{\alpha, \kappa}$ are the $T$ means with non-increasing sequence $\left\{q_{k}: k \geq 0\right\}$, then the proof of this corollary is direct consequence of Theorem 1.

Proof of Corollary 2. According to Theorem 1 a) and Proposition 2 we also have weak $(1,1)$ type inequality and by well-known density argument due to Marcinkiewicz and Zygmund [15] we have $T_{n}^{\kappa} f \rightarrow f$, a.e., for all $f \in L_{1}$. Which follows proof of Corollary 2.

Proof of Corollary [3. Since $R_{n}^{\kappa}, U_{n}^{\alpha, \kappa}$ and $V_{n}^{\alpha, \kappa}$ are the $T$ means with non-increasing sequence $\left\{q_{k}: k \geq 0\right\}$, then the proof of this corollary is direct consequence of Corollary 2 .

Proof of Theorem 2. Let the sequence $\left\{q_{k}: k \geq 0\right\}$ be non-decreasing. By combining (44) with (5) and using Abel transformation we get that 


$$
\begin{aligned}
\left|T_{n} f\right| & \leq\left|\frac{1}{Q_{n}} \sum_{j=1}^{n-1} q_{j} S_{j} f\right| \\
& \leq \frac{1}{Q_{n}}\left(\sum_{j=1}^{n-2}\left|q_{j}-q_{j+1}\right| j\left|\sigma_{j} f\right|+q_{n-1}(n-1)\left|\sigma_{n} f\right|\right) \\
& \leq \frac{1}{Q_{n}}\left(\sum_{j=1}^{n-2}-\left(q_{j}-q_{j+1}\right) j-q_{n-1}(n-1)+2 q_{n-1}(n-1)\right) \sigma^{*} f \\
& \leq \frac{1}{Q_{n}}\left(2 q_{n-1}(n-1)-Q_{n}\right) \sigma^{*} f \leq c \sigma^{*} f
\end{aligned}
$$

so that

$$
T^{*} f \leq c \sigma^{*} f .
$$

If we apply (23) and Theorem W1 we can conclude that the maximal operators $T^{*}$ is bounded from the Hardy space $H_{1 / 2}$ to the space weak $-L_{1 / 2}$. According to Proposition 2 we can conclude that $T^{*}$ has weak type- $(1,1)$ and by well-known density argument due to Marcinkiewicz and Zygmund [15] we also have $T_{n} f \rightarrow f$, a.e..

To prove part b) of theorem 2 we use the martingale, defined by (14) where $\alpha_{k}$ satisfy conditions (11-13). It is easy to show that for every non-increasing sequence $\left\{q_{k}: k \geq 0\right\}$ it automatically holds that $q_{2^{\alpha_{k}+1}} / Q_{2^{\alpha_{k}+2}} \geq c / 2^{\alpha_{k}}$. According to (16-20) we can conclude that

$$
\left|T_{2^{\alpha_{k}+2}}^{\kappa} f\right| \geq|I I|-|I| \geq \frac{2^{\alpha_{k}(1 / p-2)}}{16 \alpha_{k}} .
$$

Analogously to (21) and (22) we then get that $\sup _{k \in \mathbb{N}}\left\|T_{2^{\alpha} k+2}^{\kappa} f\right\|_{L_{p, \infty}}=\infty$ and the proof is complete.

Proof of Corollary 4. Since $B^{\alpha, \beta, *, \kappa}$ are the $T$ means with non-decreasing sequence $\left\{q_{k}: k \geq 0\right\}$, then the proof of this corollary is direct consequence of Theorem 2 .

Proof of Corollary 5. According to Proposition 2 we can conclude that $T^{*, \kappa}$ has weak type- $(1,1)$ and by well-known density argument due to Marcinkiewicz and Zygmund [15] we also have $T_{n}^{\kappa} f \rightarrow f$, a.e.. Which follows proof of Corollary 5 .

Proof of Corollary 6. Since $B^{\alpha, \beta, *, \kappa}$ are the $T$ means with non-decreasing sequence $\left\{q_{k}: k \geq 0\right\}$, then the proof of this corollary is direct consequence of Corollary 5 .

\section{REFERENCES}

[1] L. Baramidze, L. E. Persson, G. Tephnadze and P. Wall, Srtong summability and Boundedness of Maximal operators of Vilenkin-Nörlund means with non-increasing coefficients, J. Inequal. Appl., 2016, DOI: $10.1186 / \mathrm{s} 13660-016-1182-1$.

[2] I. Blahota, K. Nagy, Approximation by $\Theta$-means of Walsh-Fourier series, Anal. Math., 441 (2018) 57-71.

[3] I. Blahota, K. Nagy, G. Tephnadze, Approximation by Marcinkiewicz $\Theta$-means of double Walsh-Fourier series, Math. Inequal. Appl., 22, 3 (2019) 837-853.

[4] I. Blahota, G. Gát, Norm summability of Nörlund logarithmic means on unbounded Vilenkin groups, Anal. Theory Appl., 24 (2008), no. 1, 1-17.

[5] I. Blahota, G. Tephnadze, On the $(C, \alpha)$-means with respect to the Walsh system, Anal. Math., 40 (2014), 161-174. 
[6] I. Blahota, G. Tephnadze, Strong convergence theorem for Vilenkin-Fejér means, Publ. Math. Debrecen, 85 (1-2) (2014), 181-196.

[7] I. Blahota, L. E. Persson and G. Tephnadze, On the Nörlund means of Vilenkin-Fourier series, Czechoslovak Math. J. 65 (2015), no.4, 983-1002.

[8] G. Gát, Investigations of certain operators with respect to the Vilenkin system, Acta Math. Hungar., 61 (1993), no. 1-2, 131-149.

[9] G. Gát, On $(C, 1)$ summability of integrable functions with respect to the Walsh-Kaczmarz system, Studia Math., 130 (1998), no. 2, 135-148.

[10] G. Gát, U. Goginava, A weak type inequality for the maximal operator of $(C, \alpha)$-means of Fourier series with respect to the Walsh-Kaczmarz system, Acta Math. Hungar., 125 (2009), no. 1-2, 65-83.

[11] U. Goginava, The maximal operator of the Fejér means of the character system of the $p$-series field in the Kaczmarz rearrangement, Publ. Math. Debrecen, 71 (2007), no. 1-2, 43-55.

[12] U. Goginava, K. Nagy, On the maximal operator of $(C, \alpha)$-means of Walsh-Kaczmarz-Fourier series, Ukrainian Math. J., 62 (2010), no. 2, 175-185.

[13] N. Gogolashvili, K. Nagy, G. Tephnadze, Strong convergence theorem for Walsh-Kaczmarz-Fejér means, Mediterr. J. Math., 2021, 18 (2), 37.

[14] D. Lukkassen, L.-E. Persson, G. Tephnadze, G. Tutberidze, Some inequalities related to strong convergence of Riesz logarithmic means of Vilenkin-Fourier series, J. Inequal. Appl., 2020, DOI: https://doi.org/10.1186/s13660-020-02342-8.

[15] I. Marcinkiewicz and A. Zygmund, On the summability of double Fourier series, Fund. Math. 32 (1939), $112-132$.

[16] N. Memić, L. E. Persson, G. Tephnadze, A note on the maximal operators of Vilenkin-Nörlund means with non-increasing coefficients, Stud. Sci. Math. Hung., 53, 4, (2016) 545-556.

[17] K. Nagy, U. Goginava, Maximal operators of Walsh-Kaczmarz logarithmic means, Complex Var. Elliptic Equ., 58 (2013), no. 9, 1173-1182.

[18] C. N. Moore, Summable series and convergence factors, Dover Publications, Inc., New York 1966.

[19] F. Móricz, A. H. Siddiqi, Approximation by Nörlund means of Walsh-Fourier series, J. Approx. Theory, 70 (1992), no. 3, 375-389.

[20] K. Nagy, Approximation by Cesàro means of negative order of Walsh-Kaczmarz-Fourier series, East J. Approx., 16 (2010), no. 3, 297-311.

[21] K. Nagy, Approximation by Nörlund means of quadratical partial sums of double Walsh-Fourier series, Anal. Math., 36 (2010), no. 4, 299-319.

[22] K. Nagy, Approximation by Nörlund means of double Walsh-Fourier series for Lipschitz functions, Math. Inequal. Appl., 15 (2012), no. 2, 301-322.

[23] K. Nagy, G. Tephnadze, Approximation by Walsh-Kaczmarz-Marcinkiewicz means on the Hardy space $H_{2 / 3}$, Bulletin of TICMI, 18, 1 (2014), 110-121.

[24] K. Nagy, G. Tephnadze, On the Walsh-Marcinkiewicz means on the Hardy space, Cent. Eur. J. Math., 12,8 (2014), 1214-1228.

[25] K. Nagy, G. Tephnadze, Approximation by Walsh-Marcinkiewicz means on the Hardy space, Kyoto J. Math., 54, 3 (2014), 641-652.

[26] K. Nagy, G. Tephnadze, Kaczmarz-Marcinkiewicz means and Hardy spaces, Acta math. Hung., 149, 2 (2016), 346-374.

[27] K. Nagy, G. Tephnadze, Strong convergence theorem for Walsh-Marcinkiewicz means, Math. Inequal. Appl., 19, 1 (2016), 185-195.

[28] L. E. Persson, G. Tephnadze and P. Wall, Maximal operators of Vilenkin-Nörlund means, J. Fourier Anal. Appl., 21, (2015), no. 1, 76-94.

[29] L. E. Persson, G. Tephnadze, P. Wall, On the Nörlund logarithmic means with respect to Vilenkin system in the martingale Hardy space $H_{1}$, Acta math. Hung., 154, 2 (2018) 289-301.

[30] L. E. Persson, G. Tephnadze, G. Tutberidze, On the boundedness of subsequences of Vilenkin-Fejér means on the martingale Hardy spaces, operators and matrices, 14, 1 (2020), 283-294.

[31] L. E. Persson, G. Tephnadze, G. Tutberidze, P. Wall, Strong summability result of Vilenkin-Fejér means on bounded Vilenkin groups, Ukr. Math. J., (to appear).

[32] F. Schipp, Pointwise convergence of expansions with respect to certain product systems, Anal. Math., 2 (1976), no. 1, 65-76. 
[33] F. Schipp, W. R. Wade, P. Simon, J. Pál, Walsh series. An introduction to dyadic harmonic analysis. With the collaboration of J. Pál. Adam Hilger, Ltd., Bristol, 1990.

[34] P. Simon, On the Cesáro summability with respect to the Walsh-Kaczmarz system, J. Approx. Theory, 106 (2000), no. 2, 249-261.

[35] P. Simon, Cesáro summability with respect to two-parameter Walsh systems, Monatsh. Math., 131 (2000), no. 4, 321-334.

[36] V. A. Skvorcov, On Fourier series with respect to the Walsh-Kaczmarz system, Anal. Math., 7 (1981), no. $2,141-150$.

[37] A. Šneľder, On series of Walsh functions with monotonic coefficients, (Russian) Izvestiya Akad. Nauk SSSR. Ser. Mat. 12, (1948) 179-192.

[38] G. Tephnadze, On the maximal operators of Kaczmarz-Nörlund means, Acta Math. Acad. Paed. Nyíreg., 31 (2015), 259-271.

[39] G. Tephnadze, On the maximal operators of Walsh-Kaczmarz-Fejér means, Period. Math. Hungar., 67 (2013), no. 1, 33-45.

[40] G. Tephnadze, Approximation by Walsh-Kaczmarz-Fejér means on the Hardy space, Acta Math., Sci. Ser. B Engl. Ed., (to appear).

[41] G. Tephnadze, Fejér means of Vilenkin-Fourier series. Studia Sci. Math. Hungar., 49 (2012), no. 1, $79-90$

[42] G. Tephnadze, The maximal operators of logarithmic means of one-dimensional Vilenkin-Fourier series. Acta Math. Acad. Paedagog. Nyházi., (N.S.) 27 (2011), no. 2, 245-256.

[43] G. Tephnadze, On the maximal operators of Kaczmarz-Nörlund means, Acta Math. Acad. Paed. Nyíreg., 31 (2015), 259-271.

[44] G. Tephnadze, G. Tutberidze, A note on the maximal operators of the Nörlund logaritmic means of Vilenkin-Fourier series, Transactions of A. Razmadze Math. Inst., 174, 1 (2020), 1070-112.

[45] G. Tutberidze, Maximal operators of $T$ means with respect to the Vilenkin system, Nonlinear Studies, $27,4,(2020), 1-11$.

[46] G. Tutberidze, Modulus of continuity and boundedness of subsequences of Vilenkin- Fejér means in the martingale Hardy spaces, Georgian Math. J., (to appear).

[47] W. S. Young, On the a.e. convergence of Walsh-Kaczmarz-Fourier series, Proc. Amer. Math. Soc., 44 (1974), 353-358.

[48] F. Weisz, Martingale Hardy spaces and their applications in Fourier analysis, Lecture Notes in Mathematics, 1568, Springer-Verlag, Berlin, 1994.

[49] F. Weisz, Summability of multi-dimensional Fourier series and Hardy spaces, Mathematics and its Applications, 541, Kluwer Academic Publishers, Dordrecht, 2002.

[50] F. Weisz, $\theta$-summability of Fourier series. Acta Math. Hungar., 103 (2004), no. 1-2, 139-175.

[51] F. Weisz, $\theta$-summation and Hardy spaces. J. Approx. Theory, 107 (2000) 121-142.

[52] F. Weisz, Several dimensional $\theta$-summability and Hardy spaces. Math. Nachr., 230 (2001) 159-180.

[53] F. Weisz, Marcinkiewicz- $\theta$-summability of double Fourier series, Annales Univ. Sci. Budapest., Sect. Comp. 24 (2004) 103-118.

[54] F. Weisz, Marcinkiewicz- $\theta$-summability of Fourier transforms. Acta Math. Hungar., 96, 1-2, (2002) 149-160.

G. Tephnadze, The University of Georgia, School of science and technology, 77a Merab Kostava St, Tbilisi 0128, Georgia.

Email address: g.tephnadze@ug.edu.ge

N. Gogolashvili, The University of Georgia, School of science and technology, 77a Merab Kostava St, Tbilisi 0128, Georgia and Department of Computer Science and Computational Engineering, Uit - The Arctic University of Norway, P.O. Box 385, N-8505, NARVIK, NORWAY.

Email address: nata.gogolashvili@gmail.com 